Research Article

\title{
Emissions of Airport Monitoring with Solar Occultation Flux-Fourier Transform Infrared Spectrometer
}

\author{
Xin Han, ${ }^{1,2}$ Xiangxian Li ${ }^{D},{ }^{1}$ Minguang Gao, ${ }^{1}$ Jingjing Tong, ${ }^{1}$ Xiuli Wei, ${ }^{1}$ Sheng Li, \\ Shubin Ye, ${ }^{2,3}$ and Yan $\mathrm{Li}^{1,2}$ \\ ${ }^{1}$ Anhui Institute of Optics and Fine Mechanics, Chinese Academy of Sciences, Hefei 230026, China \\ ${ }^{2}$ University of Science and Technology of China, Hefei 230026, China \\ ${ }^{3}$ Hefei Institutes of Physical Sciences, Chinese Academy of Sciences, Hefei 230031, China
}

Correspondence should be addressed to Xiangxian Li; lixx@aiofm.ac.cn

Received 6 November 2017; Revised 28 January 2018; Accepted 11 February 2018; Published 18 April 2018

Academic Editor: Feride Severcan

Copyright (C) 2018 Xin Han et al. This is an open access article distributed under the Creative Commons Attribution License, which permits unrestricted use, distribution, and reproduction in any medium, provided the original work is properly cited.

\begin{abstract}
Both domestic and international aviation industries have experienced a boom, which results in a dramatic increase in emissions of the aviation industry in recent decades. Therefore, domestic and abroad scientists adopted different methods to measure emissions; however, there are no appropriate methods to measure the emissions of the whole airport. In order to provide data support for the relevant departments to take appropriate emission reduction measures, solar occultation flux-Fourier transform infrared spectrometer (SOF-FT-IR) is used to monitor the emissions of Beijing Capital International Airport. $\mathrm{CO}, \mathrm{CO}_{2}, \mathrm{C}_{2} \mathrm{H}_{4}, \mathrm{and}_{\mathrm{CH}} \mathrm{O}$ are selected as the target gases and are quantitatively analyzed with the nonlinear least squares method to get the column concentration. Then, the flux can also be calculated by linking the wind velocity and direction with the column concentration. A comparison between the results measured by SOF-FT-IR and the results measured by the method published by the Intergovernmental Panel on Climate Change (IPCC) indicates that auxiliary power equipment and ground support equipment for the emission of the airport are also important emission sources besides the aircraft and the concentration distribution gives powerful and useful pieces of evidence to locate the emission sources. In order to decrease the contribution of the airport to the air pollution, the key point is to reduce the emissions of the APU and mobiles in the airport.
\end{abstract}

\section{Introduction}

With the development of technology and society, the aviation industry has experienced an unprecedented development period in recent years. Data from the International Civil Aviation Organization (ICAO) and International Air Transport Association (IATA) indicate that the total annual output of the world's civil aviation has reached $\$ 400$ billion [1] and the amount of cargo mail is also more than 41 million tons [2]. In 2004, the major projects of large aircrafts are included in the national long-term technology development plan which expresses the strong desire to develop the aviation industry of the government [3].

The predicted growth of air transport in the next few decades (particularly in Asia) indicates that emissions may grow exponentially from the current levels. It is urgent for the whole world to take effective methods to monitor and then reduce the emission of aircrafts. The exhaust emissions of aircrafts mainly are carbon oxides, nitrogen oxides, hydrocarbons, and so on. Almost all these gases have diverse impact on air quality, human health buildings, and so on. With the increase in air traffic flow in terminal areas, the aircraft has become a significant source of air pollution [4]. $\mathrm{CO}_{2}$ emitted by the aircraft accounts for $2 \%$ of the worldwide anthropogenic $\mathrm{CO}_{2}$ emission; this proportion is expected to increase to $3 \%$ by 2050 . EU has brought the $\mathrm{CO}_{2}$ emission in its carbon emission trading scheme, which means that the airlines that exceed the standard quota have to buy emission credits [5]. If the principle of "polluter pays" comes into effect, then it is necessary to have more accurate methods for dealing with the environmental effects of air transport. Current emission regulations have focused on local air quality in the vicinity of airports. The ICAO has set an environmental goal to limit and reduce the influences 
of aircraft pollutant emissions on local air quality from aircraft-related activities [6]. Therefore, it is necessary to take efficient methods to monitor the emissions of aircrafts and the whole airport. The impacts of aviation emissions on the atmosphere are shown in Figure 1 [7]. Compared to other sources, aviation emissions are a relatively small contributor to air quality concerns with regard to both local air quality and greenhouse gas emissions. Although small, however, aviation emissions cannot be ignored.

A complete flight mission can be divided into two parts: landing and take-off (LTO) phase, which is the focus of this study, and cruse phase, which is like what is shown in Figure 2 [8]. The cruse phase is the phase where the aircraft is in the latitude of $8 \mathrm{~km}-13 \mathrm{~km}$; the emissions in this phase have little impact on the human beings, so the emissions in the cruse phase is not discussed in the paper. The LTO phase defined by the ICAO (1993) includes all activities near the airport that take place below the altitude of $3000 \mathrm{ft}(1000$ meters). The LTO phase includes taxi-in and taxi-out, takeoff, climb-out, and approach-landing. Currently, modeling analysis (e.g., EDMS) and sampling analysis (e.g., FT-IR and DOAS) [9] are used to monitor aircraft emissions. Fan et al. [10] used the EDMS model to study the air quality in Beijing Capital Airport and established the emission inventory. Kesgin [11] combined the EDMS model with the method used by Woodmansey-Patterson to estimate the maximum and minimum emissions in an airport in Turkey. Sampling analysis can be implemented with an instrument in the field, that is, Fourier transform infrared spectroscopy (FT-IR) and differential optical absorption spectroscopy (DOAS), which are utilized to detect carbon oxides and nitrogen oxides; also, chromatography can be applied to measure the concentration of VOCs and CO in the laboratory. Yu et al. [12] used partition sampling methods to carry out research for Los Angeles International Airport and Hong Kong International Airport. In combination with the later nonparametric regression data, the results are obtained and showed that $\mathrm{SO}_{2}$ can be used as a tracer of airport emissions. Liu et al. [13] did research on the two sets of gases emitted by the JT3D-7 engine; the consequences of sampling analysis are consistent with the results obtained by using the direct calculation program. Schäfer et al. [14] used the FT-IR and DOAS to analyze the emissions of some parts of European airports and compared the results with the data published by the ICAO; the results indicated that the concentration of $\mathrm{CO}$ measured by FT-IR and DOAS is higher than that of the ICAO, which has the lower NOx emissions. Moreover, the emissions of different airplanes that have different engines are rather different. All these methods have their own advantages; however, these methods are only suitable for the measurement or calculation of the emissions of an aircraft; they can be used to monitor the emissions of the whole airport.

FT-IR has the advantages of fast measuring speed, high precision, high resolution, wide measuring range, large luminous flux, and low stray light; it is suitable for automatic online measurement of a variety of gaseous pollutants at the same time, especially in the atmosphere of volatile organic compounds, and it is widely used in the air pollutant monitoring. SOF-FT-IR, short for solar occultation flux-
Fourier transform infrared spectrometer, is a kind of passive monitor technology, and it regards the sun as the infrared light source. The main parts of the system are a solar tracker and a spectrometer; the former is used to induce the solar ray that has been absorbed by the pollutant gas into the spectrometer, and then the absorbed spectrum can be obtained. It has high flexibility and can be used to monitor the distribution of concentration and flux of a pollutant area.

According to the research, it can be known that most studies pay attention to emissions of a single aircraft or engine. However, the SOF-FT-IR system can measure the concentration and flux of the whole airport. The result also indicates that the system is a significant method to monitor the emissions of the aircraft and make up for the shortages of current methods.

The remainder of the paper is organized as follows: in Section 2, the theoretical analysis is presented, and in Section 3 , the experiment is described. The analysis of the emission is shown in Section 4, and conclusions are given in Section 5.

\section{Theory}

SOF-FT-IR, short for solar occultation flux-Fourier transform infrared spectrometer, is a kind of passive monitor technology, and it regards the sun as the infrared light source. The main parts of the system are a solar tracker and a spectrometer; the former is used to induce the solar ray that has been absorbed by the pollutant gas into the spectrometer, and then the absorbed spectrum can be obtained. The transmission of solar radiation is analyzed in accordance with the atmospheric transmission model, and then the nonlinear least squares method-based on BouguerLambert-Beer's law-is used to get the column concentration distribution. Besides, flux emission of target gases in the monitoring area also can be obtained in combination with meteorological information.

2.1. Atmospheric Transmission Model. When the sun transmits the energy to earth, it will be absorbed and scattered by particles in the atmosphere. Since temperature and pressure are different in different heights, the influence of radiation is not the same; the atmospheric transmission model should be followed when calculating the radiation flux model at a certain height, as shown in Figure 3(a). The received solar radiation flux of each layer is

$$
I_{i}(v)=\varepsilon_{i}(v) B_{i}\left(v, T_{i}\right)+\tau_{i}(v) I_{i-1}(v)+\beta_{i}(v) .
$$

Here, $\varepsilon_{i}(v), \tau_{i}(v)$, and $\beta_{i}(v)$ is the emissivity, transmittance, and reflectivity in the $i$-layer, respectively, $I_{i-1}(v)$ is the solar radiation intensity of the upper layer, and $B_{i}\left(v, T_{i}\right)$ is the blackbody radiation intensity when the temperature is $T_{i}$.

Compared to atmospheric absorption and transmission during the transmission, the contribution of scattering is so small that it can be ignored. Combined with Kirchhoff's law, the emissivity of the medium is equal to the absorbed rate of it at a given wavelength; the forum can be expressed as

$$
I_{i}(v)=(1-\tau(v)) B_{i}\left(v, T_{i}\right)+\tau(v) I_{i-1}(v) .
$$



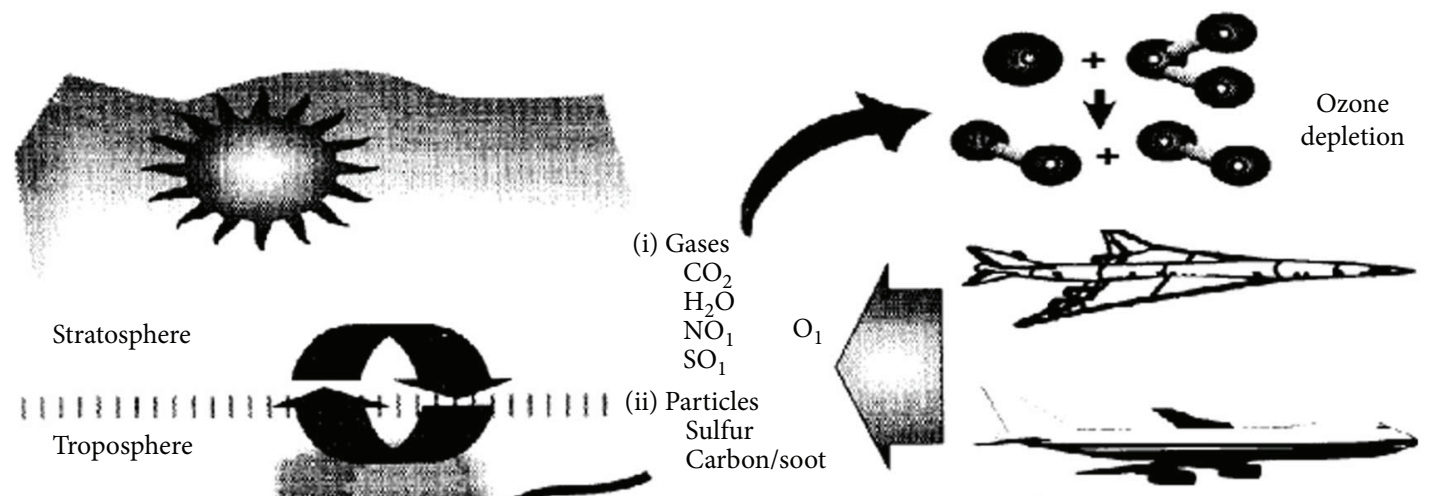

$$
\text { Stratosphere }
$$

$1111\} 11\} 111114$ Troposphere

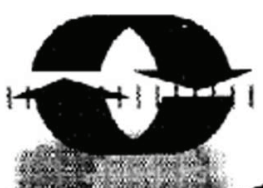

111111 (ii) Particles Carbon/soot

(iii) Cloud formation

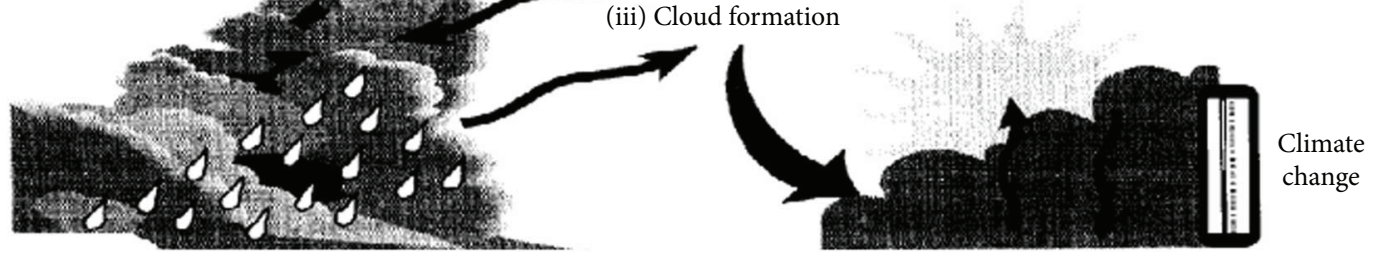

Figure 1: Aircraft emissions and the global environment.

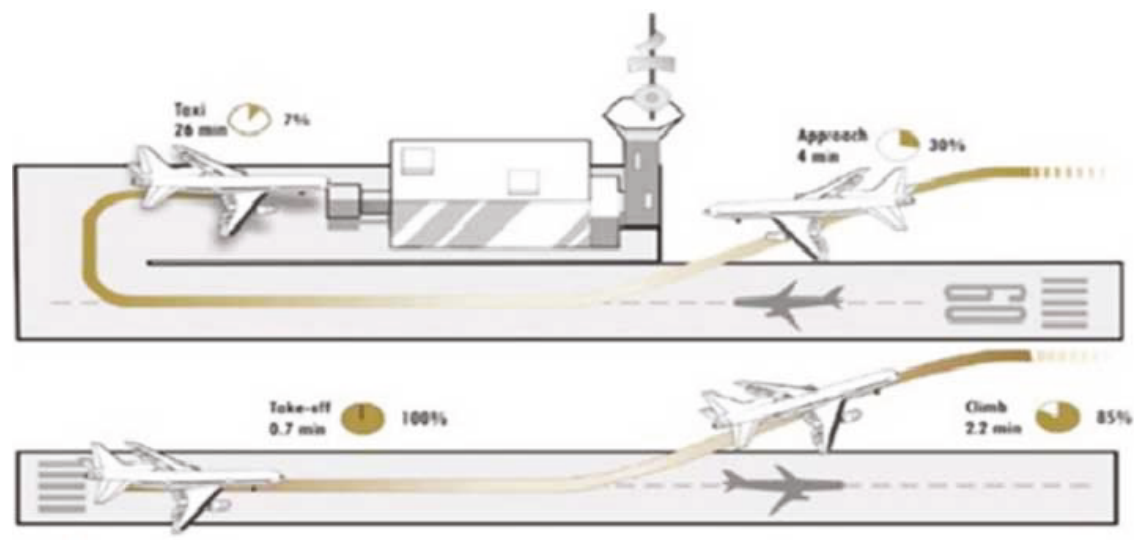

D newe renings

Figure 2: Phase of flight of an aircraft.

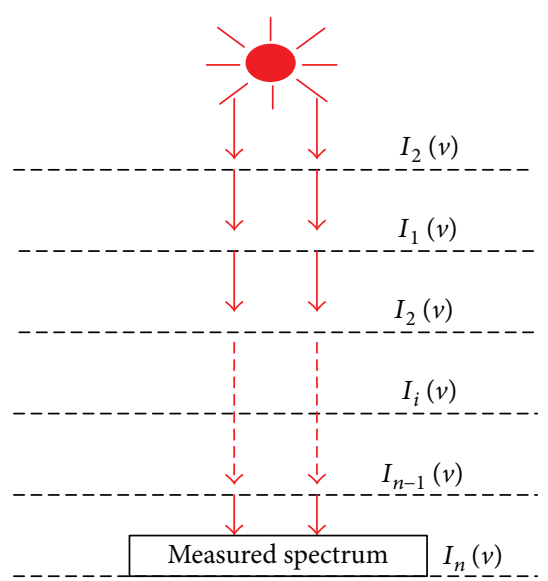

(a)

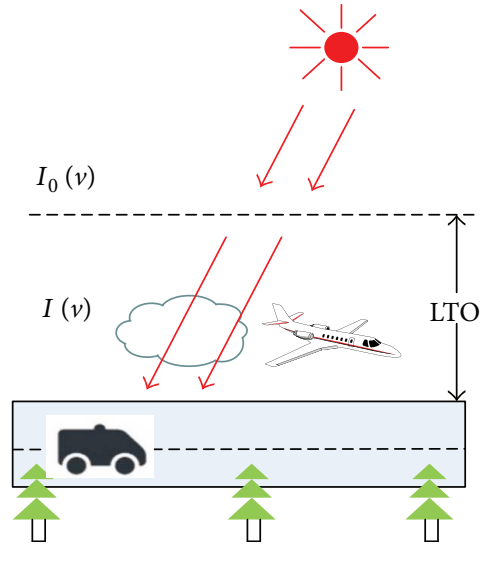

(b)

FIGURE 3: Atmospheric transmission model: (a) multilayer atmospheric transmission model and (b) two-layer atmospheric transmission model. 
As the focus of the paper is the gas concentration near the ground, the multilayer atmospheric transmission model can be simplified as the two-layer atmospheric transmission model, because the radiation of material in the near surface is much smaller than the radiation from the solar radiation of the upper layer; (2) can be simplified to

$$
I(v)=\tau(v) I_{0}(v)
$$

2.2. Beer's Law. Beer's law, which is also called BouguerLambert-Beer's law, is the basic law of the quantitative analysis of the spectra. The transmittance of a multicomponent gas mixture in the wavenumber of $v$ is equal to the ratio of the measured spectrum and the background spectrum of samples.

$$
\tau(v)=\frac{I(v)}{I_{0}^{\prime}(v)}=e^{-\alpha(v) l},
$$

where $\alpha(v)$ is the linear absorption coefficient at $v, l$ is the optical path, and $I_{0}^{\prime}(v)$ is the background spectrum which is measured in the direction of the wind of the measured path. Since the absorption of each gas is individual, the measured spectrum and background spectrum can be expressed as follows:

$$
\begin{aligned}
& I(v)=I_{0}(v) * e^{-\sum_{i} \delta_{i} c_{i} l_{i}} * e^{-\sum_{j} \delta_{j} c_{j} l_{j}}, \\
& I_{0}^{\prime}(v)=I_{0}(v) * e^{-\sum_{i} \delta_{i} c_{i} l_{i}}
\end{aligned}
$$

where $\delta_{i}$ is the conventional gas absorption interface, $c_{i} l_{i}$ is the column concentration, and $\delta_{j}$ and $c_{j} l_{j}$ is the absorption interface and column concentration of the target gas, respectively. The absorption interface can be acquired from the HITRAN database. HITRAN is an acronym for highresolution transmission molecular absorption database and is a compilation of spectroscopic parameters that a variety of computer codes use to predict and simulate the transmission and emission of light in the atmosphere. By combining the HITRAN database with the temperature and pressure, $\delta_{j}$ can be calculated.

2.3. Quantitative Analysis. Quantitative analysis of the gas is variable in accordance with the absorbance of the sample database, the spectral data and model data between the concentrations of gases. According to Beer's law, an equation can be known: $A=\alpha l c=k c$. Theoretically, only $\boldsymbol{k}$ needed to be calculated, from a practical point of view; however, it is the sun, instead of a monochromatic light, that is used as the light source; therefore, the test gas has different absorption intensities for each frequency and wave peak rather than forming a single absorption line. Secondly, when gas component concentration is high enough, the distance between molecules becomes so short that the charge distribution interacted with each other. The interaction will result in the change of absorption coefficient. The simple linear function is unable to describe the relationship between absorbance and concentration.
Therefore, nonlinear relationship between concentration and absorbance only can be solved through the establishment of a nonlinear model. The common nonlinear models are the nonlinear least squares (NLLS) method, artificial neural network (ANN), support vector machine (SVM), etc. [15]. In this paper, NLLS is utilized to get the calculated spectrum which is used to fit the measure spectrum to give the concentration of target gases. For nonlinear function, it usually puts Taylor series expansion and successive linearization and then completed the calculation with the iterative solver.

The difference between the measured spectrum and the background spectrum actually is the atmospheric transmittance which superimposed an $\boldsymbol{n}$-order polynomial that changes with the wavenumber $v$ slowly:

$$
B(v)=b_{0}+b_{1} v+b_{2} v^{2}+b_{3} v^{3}+\cdots+b_{n} v^{n}
$$

where the polynomial coefficients $b_{i}(i=1,2, \ldots, n)$, the change of column concentration of common gases $\Delta\left(c_{i} l_{i}\right)$, and the column concentration $c_{j} l_{j}$ of target gases are pending. The transmittance iterative calculation can be expressed as

$$
\tau^{\prime}(v)=\left(b_{0}+b_{1} v+\cdots+b_{n} v^{n}\right) e^{-\sum_{j} \sigma_{j}(v) \Delta\left(c_{i}^{\prime} l_{j}^{\prime}\right)} e^{-\sum_{j} \sigma_{j}(v) c_{j}^{\prime} l_{j}^{\prime}}
$$

According to the change of the baseline of measure spectrum and ratio spectrum, the appropriate $\boldsymbol{n}$ should be determined. For the majority of interesting problems, $n=2$ or 3 .

The squared error is the sum of the squares of the difference between the computed spectrum and the measured spectrum, and one would reduce the error by iterating $b_{i}, \Delta$ $\left(c_{i} l_{i}\right)$, and $c_{j} l_{j}$ to obtain the minimum, with the $c_{j} l_{j}$ being the column concentration of the target gas at that time.

$$
\gamma^{2}=\sum_{v}\left(\tau(v)-\tau^{\prime}(v)\right)^{2}=\min .
$$

Since the column concentration is known, the flux velocity can be computed in the light of the following formula:

$$
\text { flux }=\int_{X 1}^{X 2} C_{l i}(x) u(x) \cos \theta(x) d x .
$$

Here, $X_{1}$ and $X_{2}$ are the start position and end position of the monitoring path, respectively, $x$ is the measured point in the monitoring path, $u(x)$ is the wind velocity, $\theta(x)$ is the angle between the direction that the monitoring vehicle is traveling and the wind direction, and $C_{l i}(x)$ represents the column concentration at $x$ point.

$$
C_{l i}(x)=C_{i}(x) L_{i}(x) \sin (\alpha(x))
$$

where $a(x)$ is the elevation angle of the sun; the value of these parameters can be calculated with the GPS module.

$$
\sin \alpha=\sin \varphi \sin \delta+\cos \varphi \cos \delta \cos \omega,
$$

where $\delta$ is the declination angle $\left(\delta=23.45^{*} \sin \left(360^{*}(284+\right.\right.$ $n) / 365)$, where $n$ is the first few days of the year), $\omega$ is the hour angle $(\omega=(T-12) * 15)$, and $\varphi$ is the latitude. 


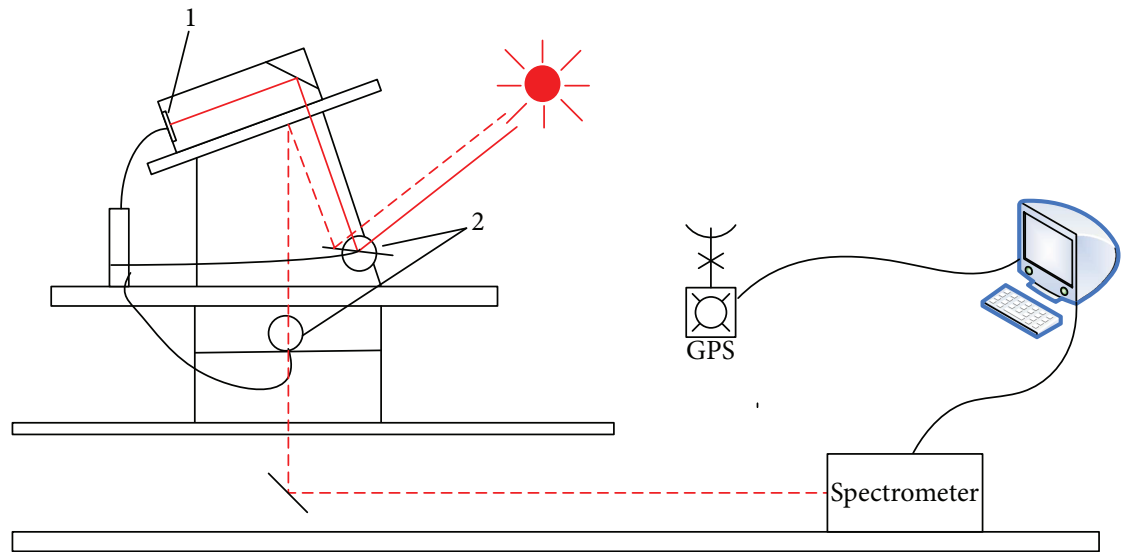

FIgURE 4: The schematic of SOF-FT-IR.

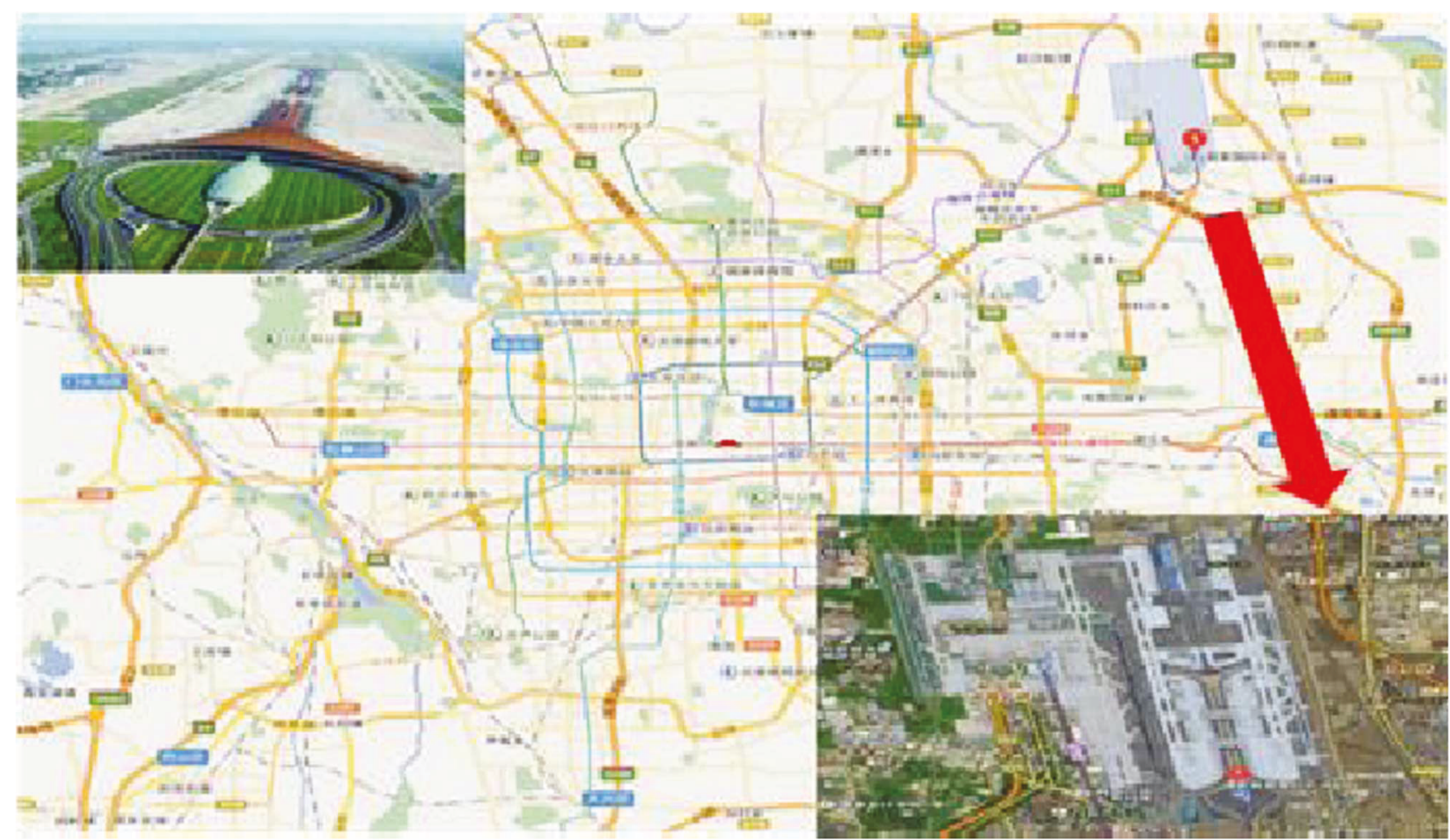

Figure 5: Beijing Capital International Airport.

\section{Experiment}

3.1. Experimental Device. SOF-FT-IR is firstly put forward by Chalmers University in Sweden; it is the instrument which combined the solar tracker and spectrometer to monitor the emissions in the chemical plant zone or anywhere else. The whole system is placed in a car during the monitor process; the schematic of which is shown in Figure 4. The PSD module (indicated by 1 ) is used to detect the position of the sun and then input the position signal to the microcontroller to control the motors - indicated by 2 in Figure 4 - and then rotate the mirrors at a certain angle to ensure that the spectrometer obtains the maximum light intensity.
3.2. Experimental Site. To a certain degree, the busier an airport is, the more emissions it has. Therefore, in this experiment, Beijing Capital International Airport which is the busiest airport in China is selected as the experimental site.

Beijing Capital International Airport (ICAO airport code: ZBAA; IATA code: PEK), located in Shunyi District, Beijing, China, is the busiest international airport in China. It was built in 1958 which has operated for more than 50 years, and the passengers throughput have increased from 1.03 million in 1978 to 8.9 million in 2015; meanwhile, the freight also increases to 1.848 million tons by 2015 and shows a sustained growth trend. The position of the experimental site is illustrated in Figure 5. 


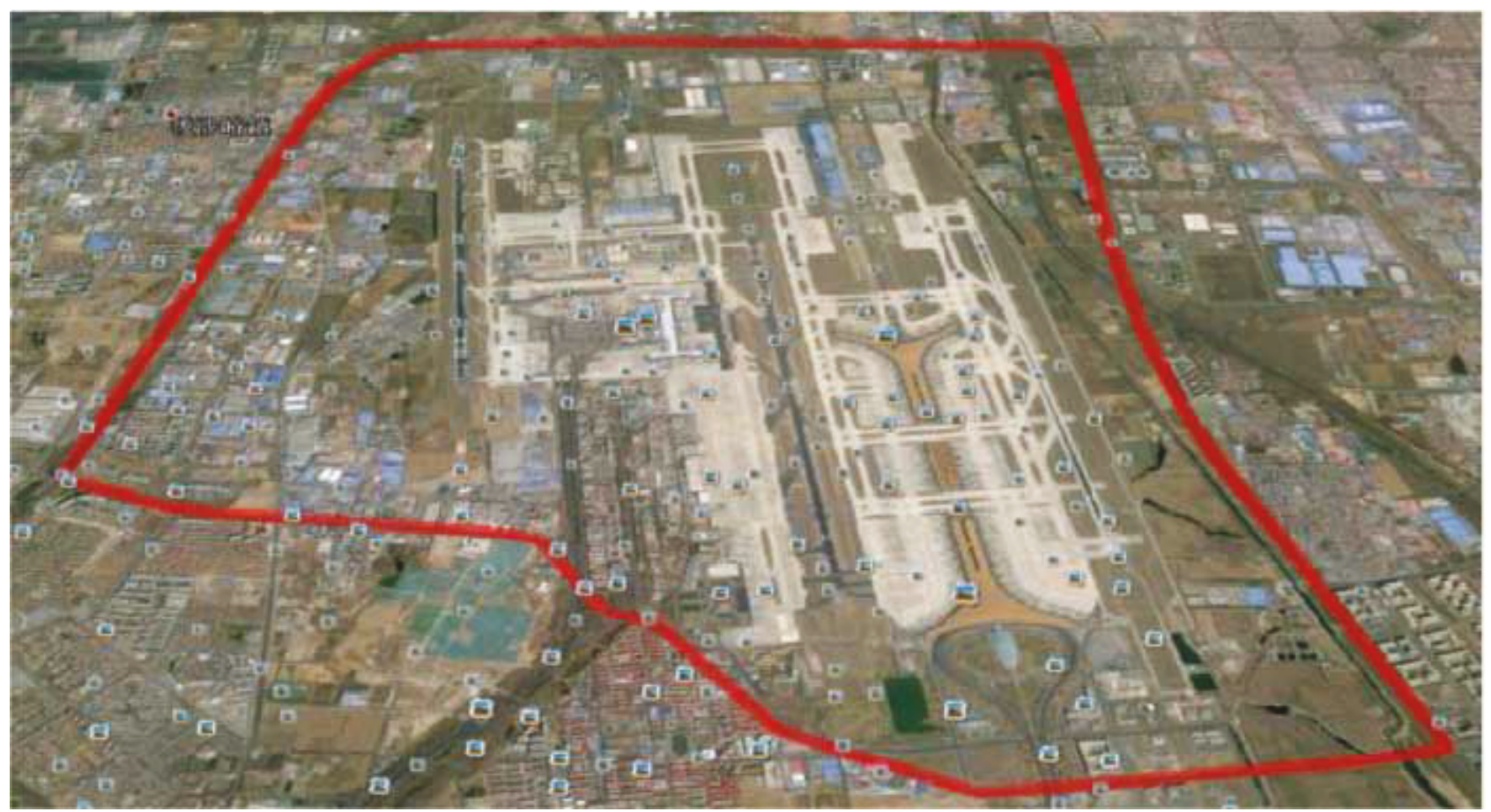

FIGURE 6: Monitoring path.

TABLE 1: Measurement time and effective circles per day.

\begin{tabular}{lcc}
\hline Measurement phase & Measurement time & Effective circles \\
\hline Winter & $2014 / 12 / 10 \sim 2014 / 12 / 16$ & 9 \\
Summer & $2015 / 5 / 12 \sim 2015 / 5 / 16$ & 10 \\
Autumn & $2015 / 8 / 21 \sim 2015 / 8 / 29$ & 5 \\
Spring & $2015 / 12 / 4 \sim 2015 / 12 / 05$ & 6 \\
\hline
\end{tabular}

In accordance with the traffic environment around the airport, the monitoring path was selected and shown in Figure 6. The entire measurement path is a closed loop and its total length is about $27 \mathrm{~km}$, and the measurement is operated in four seasons. The numbers of measured circles are different every day. Some data should be deleted based on actual situation before analyzing the data; the measurement time and the effective number of circles are shown in Table 1.

3.3. Experimental Data. As spectral broadening and instrument line shape function will affect the measured spectra, the background spectrum is measured in the upwind direction of the measurement path as a reference to minimize the impact of these effects on the inversion result of the measurement process. Figure 7 shows the typical background spectrum in four seasons.

With the related information, it is known that the main emissions of the airport are carbon oxides, nitrogen oxides, and hydrocarbons. $\mathrm{CO}_{2}, \mathrm{CO}, \mathrm{C}_{2} \mathrm{H}_{4}$, and $\mathrm{CH}_{2} \mathrm{O}$ are selected as target gases through continuous debugging, and the bands in Table 2 are used to quantitatively inverse their concentration, respectively. A typical measured spectrum is shown in Figure 8.
The whole process of inversion is the extraction of the parameter from the database which participates in the calculation, and then the calculated spectrum and the measured spectrum are fitted to give the concentrations of the target gases. The better the effect of fitting between the measured spectrum and the calculated spectrum is, the smaller the error is, which also suggests more accurate result. The fitting results are shown in Figure 9.

It can be done in two ways to analyze the experimental data: the concentration distribution and flux of target gases, respectively. The relationship between the concentration distribution and the wind velocity and wind direction at four seasons is talked about at first. With the information of the GPS module and wind velocity and direction, the distribution in the monitoring path is known; the typical distribution in four seasons are shown in Figure 10.

In Figure 10, the red arrow denotes the wind direction. The figure shows that the concentration distribution is substantially in line with the feature of low concentration in the upwind direction and high concentration in the downwind direction. Some points in the direction of the wind also have the higher concentration point; it is mainly because the uncertainties of wind velocity and direction and the bump or anything else happened in the monitoring vehicle in the measurement process. According to the distribution of concentration, we can locate the emission sources immediately.

\section{Total Flux Estimates}

In 2006 IPCC Guidelines for National Greenhouse Gas Inventories, two methods are put forward to estimate the emission of the aircraft. Method 1 is based on the fuel, and the formula is 

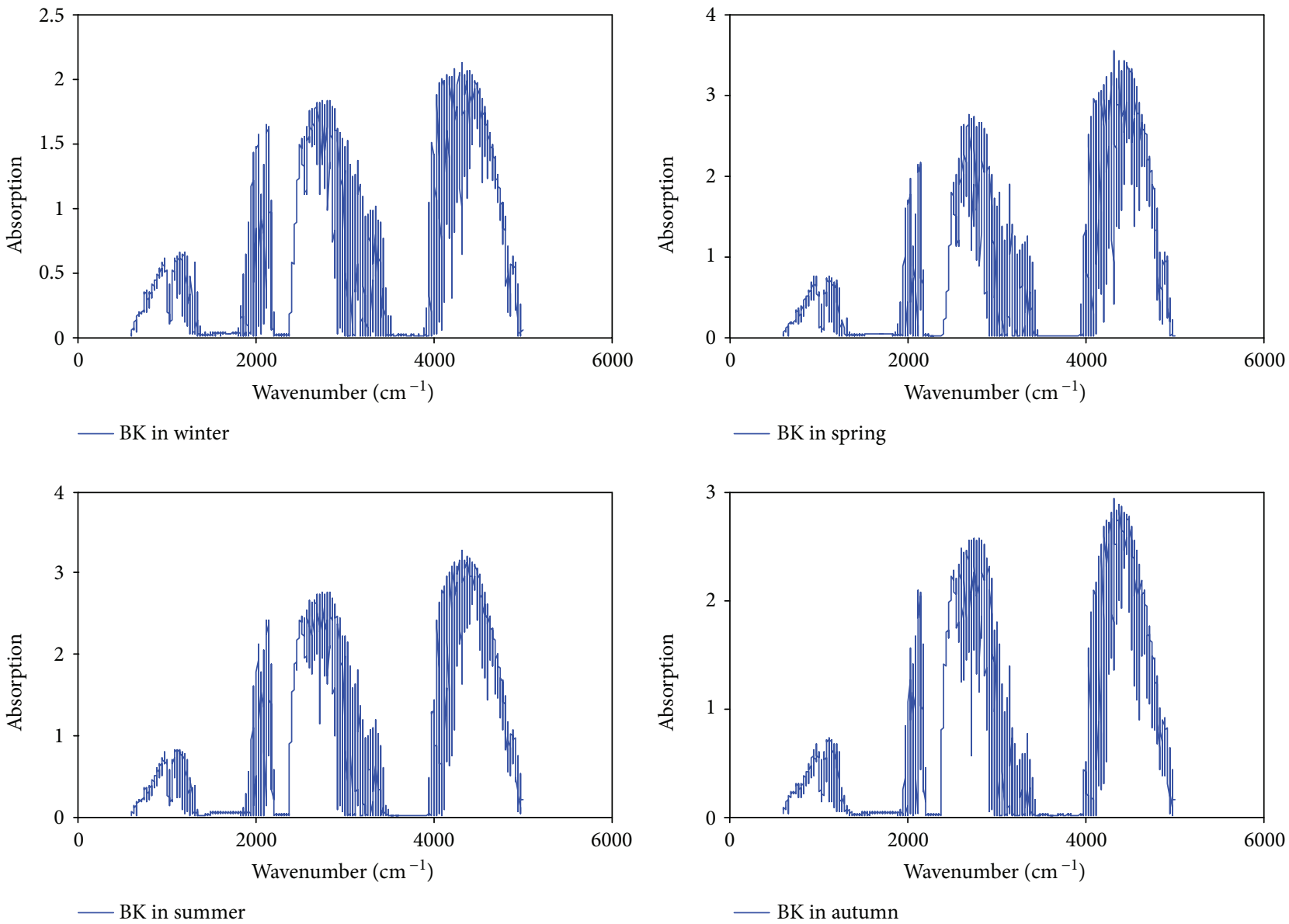

Figure 7: Background spectrum at four seasons.

TABLE 2: Target gases and their inversion bands.

\begin{tabular}{lc}
\hline Target gases & Inversion bands $\left(\mathrm{cm}^{-\mathbf{1}}\right)$ \\
\hline $\mathrm{CO}_{2}$ & $2080 \sim 2200$ \\
$\mathrm{CO}$ & $2080 \sim 2200$ \\
$\mathrm{C}_{2} \mathrm{H}_{4}$ & $880 \sim 960$ \\
$\mathrm{CH}_{2} \mathrm{O}$ & $2670 \sim 2800$ \\
\hline
\end{tabular}

$$
f=k * e,
$$

where $\boldsymbol{f}$ is the flux, $\boldsymbol{k}$ is the total amount of the consumed fuel per year, and $\boldsymbol{e}$ is the emission factor per kilogram fuel.

The second method takes into account both the numbers of take-off and landing cycles, also taking into account fuel consumption. In this experiment, the concentration and flux are measured below $1000 \mathrm{~m}$; therefore, the emission of the LTO phase is enough; that is,

$$
F=N * E_{\mathrm{LTO}} .
$$

where $N$ is the landing number in one year and $E_{\text {LTO }}$ is the emission factor in one LTO phase.

As the detailed information such as landing number and emission factor in each season is inaccessible to us, then the total emissions in the LTO phase in 2015 are estimated in this paper. By studying the related information, it is known that total landing number of Beijing Capital Airport in 2015 is 590169; the domestic aircraft and the international aircraft are Df and If, respectively, which have a total landing number of 457572 and 132597, respectively [16]. Here, the second method is used to estimate the flux.

4.1. Method 2. The emission factor in the LTO phase is shown in Table 3 [4].

Substituting these parameters into (14), the flux can be expressed as follows:

$$
\begin{aligned}
E\left(\mathrm{CO}_{2}\right)= & \mathrm{EI}_{\mathrm{D}}\left(\mathrm{CO}_{2}\right) * \mathrm{Df}+\mathrm{EI}_{\mathrm{I}}\left(\mathrm{CO}_{2}\right) * \mathrm{If}=457572 \\
& * 2600+132597 * 5094=1.865 * 109 \mathrm{~kg}, \\
E(\mathrm{CO})= & \mathrm{EI}_{\mathrm{D}}(\mathrm{CO}) * \mathrm{Df}+\mathrm{EI}_{\mathrm{I}}(\mathrm{CO}) * \mathrm{If}=457572 \\
& * 11.8+132597 * 6.1=6.208 * 106 \mathrm{~kg}, \\
E(\mathrm{NM}-\mathrm{VOCs})= & \mathrm{EI}_{\mathrm{D}}(\mathrm{NM}-\mathrm{VOCs}) * \mathrm{Df}+\mathrm{EI}_{\mathrm{I}}(\mathrm{NM}-\mathrm{VOCs}) \\
& * \mathrm{If}=457572 * 1.15+132597 \\
& * 0.54=5.978 * 105 \mathrm{~kg} .
\end{aligned}
$$

Flux velocity (FV) can be computed with SOF-FT-IR; meanwhile, once there is a big emission that happened out 

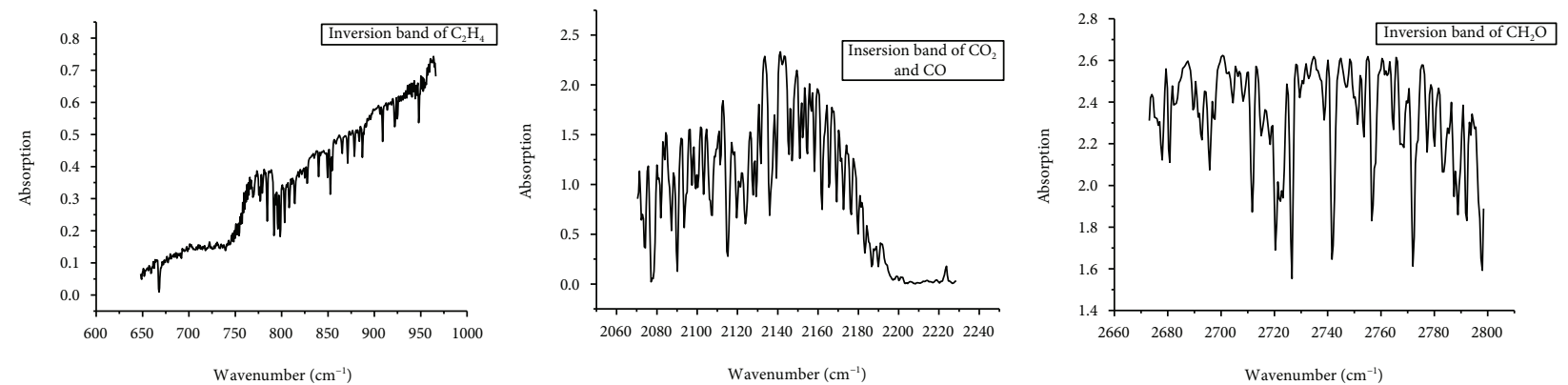

FIGURE 8: Inversion bands of target gases.

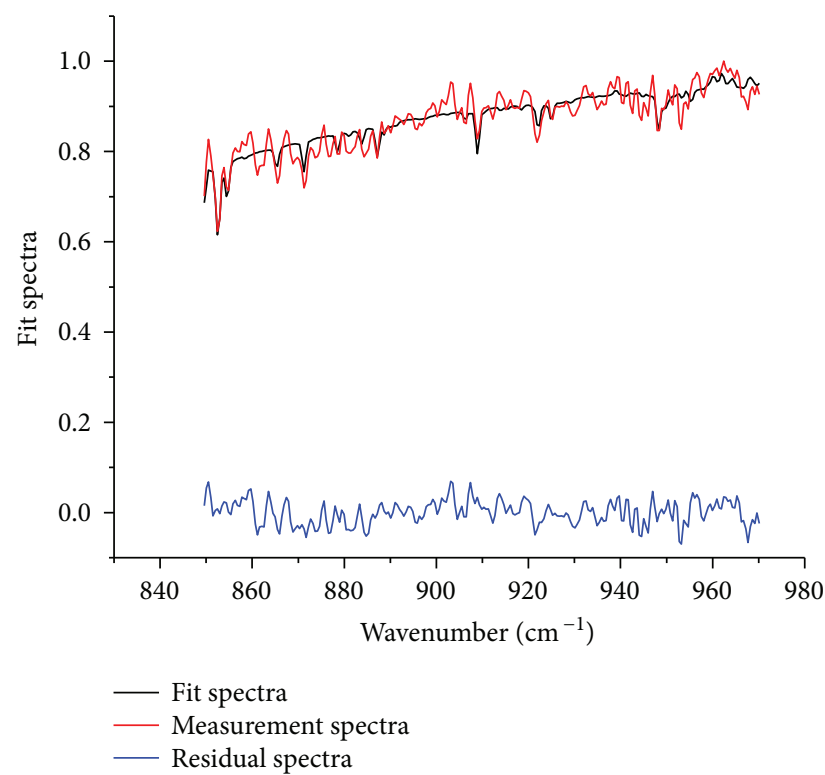

(a)

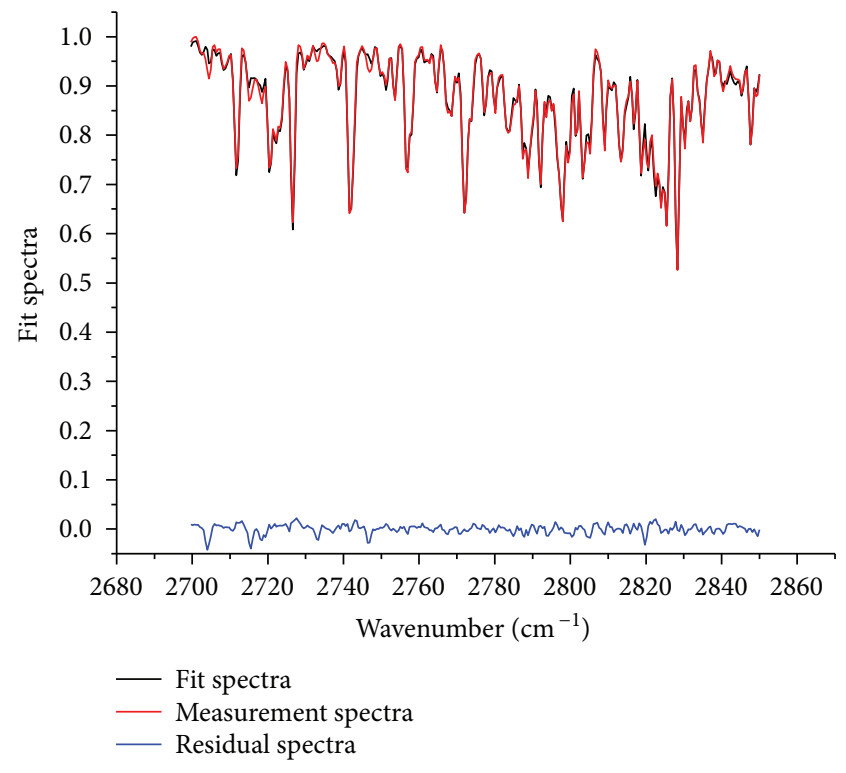

(b)

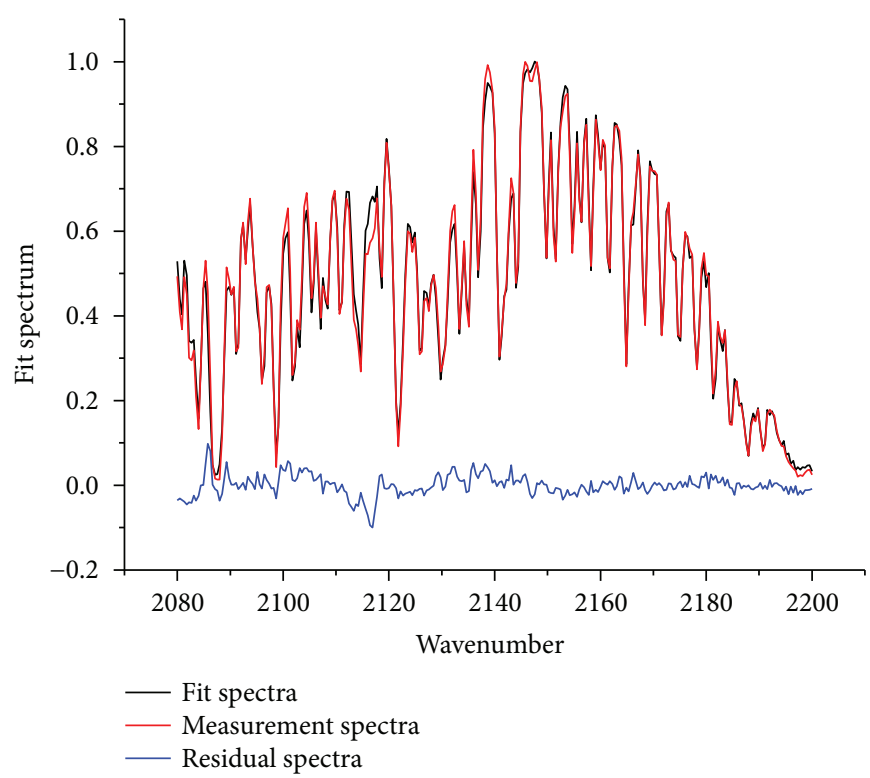

(c)

FIGURE 9: The fitting result of target gases: (a) the fitting result of $\mathrm{C}_{2} \mathrm{H}_{4}$, (b) the fitting result of $\mathrm{CH}_{2} \mathrm{O}$, and (c) the fitting result of $\mathrm{CO}$ and $\mathrm{CO}$. 

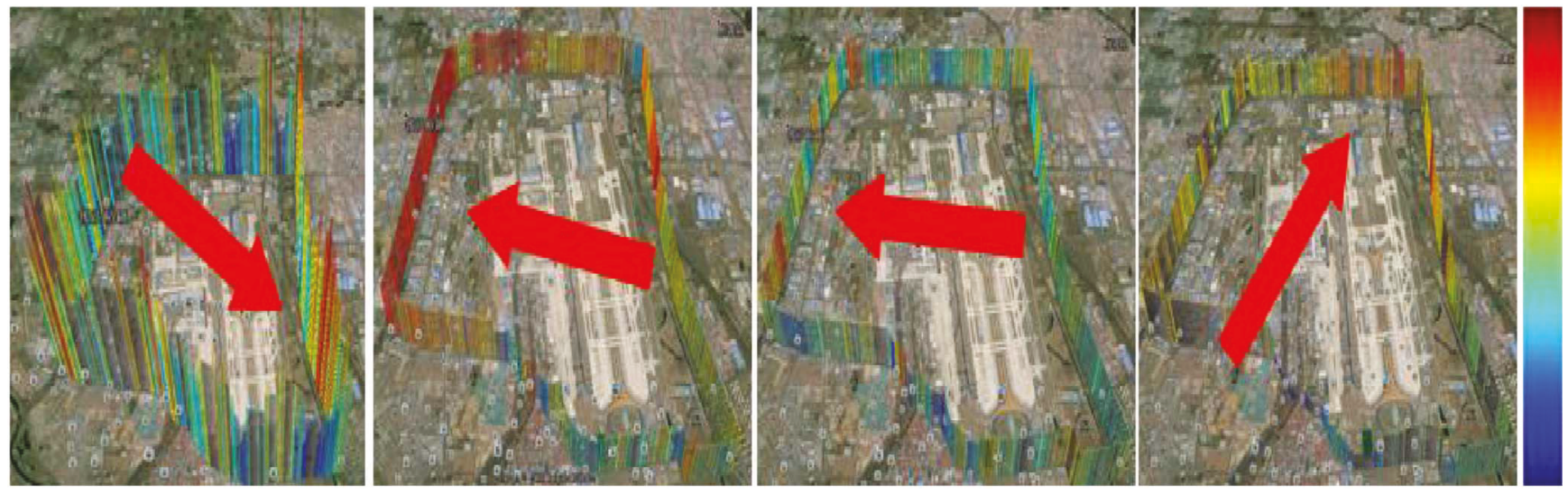

Figure 10: Typical concentration distribution in four seasons.

TABLE 3: The emission factor of $\mathrm{CO}_{2}$, CO, and NM-VOCs.

\begin{tabular}{lccc}
\hline EI $(\mathrm{kg} / \mathrm{LTO})$ & $\mathrm{EI}(\mathrm{CO} 2)$ & EI $(\mathrm{CO})$ & EI (NM-VOCs) \\
\hline Domestic & 2600 & 11.8 & 1.15 \\
International & 5094 & 6.1 & 0.54 \\
\hline
\end{tabular}

of the monitoring path, a negative value of flux velocity may happen which has a serious impact on the estimation of flux of Beijing Capital Airport. It is unreasonable to use the average emission flux rate to calculate the flux. By taking into account the specific circumstances of the measurement period and decreasing the influence of wind and other weather parameters, the data were measured in the time when there is no wind or the wind is so small that it almost can be ignored. The appropriate rate of $\mathrm{CO}_{2}$ emission flux is shown in Table 4.

The flux calculated with SOF-FT-IR of Beijing Capital Airport is

$$
\begin{aligned}
E_{\mathrm{sof}}\left(\mathrm{CO}_{2}\right) & =\mathrm{FV}\left(\mathrm{CO}_{2}\right) * M\left(\mathrm{CO}_{2}\right) * 24 * 365=9.361 * 10^{9} \mathrm{~kg}, \\
E_{\mathrm{sof}}(\mathrm{CO}) & =\mathrm{FV}(\mathrm{CO}) * M(\mathrm{CO}) * 24 * 365=2.623 * 10^{7} \mathrm{~kg}, \\
E_{\mathrm{sof}}\left(\mathrm{C}_{2} \mathrm{H}_{4}\right) & =\mathrm{FV}\left(\mathrm{C}_{2} \mathrm{H}_{4}\right) * M\left(\mathrm{C}_{2} \mathrm{H}_{4}\right) * 24 * 365=7.67 * 10^{5} \mathrm{~kg}, \\
E_{\mathrm{sof}}\left(\mathrm{CH}_{2} \mathrm{O}\right) & =\mathrm{FV}\left(\mathrm{CH}_{2} \mathrm{O}\right) * M\left(\mathrm{CH}_{2} \mathrm{O}\right) * 24 * 365=8.73 * 10^{5} \mathrm{~kg} .
\end{aligned}
$$

The results indicate that the flux measured with SOF-FTIR is much higher than the data calculated with method 2, which is mainly because the data calculated with method 2 is the emission flux of the main engine of the aircraft while the emission flux measured with SOF-FT-IR are that of the whole airport.

Peace et al. [17] point out that the emission inventory published by the ICAO does not take into account the spread of pollutants in the atmosphere. They have chosen the road traffic and aircraft emissions as a line source, the chemical plant near the airport as a point of power, and the auxiliary power equipment and ground support equipment and other point emissions as point source emissions, and the relationship between the emission of the airport and the other emission sources is known. Xia [18] analyzed the influence of
TABLE 4: Typical flux value.

\begin{tabular}{lc}
\hline Components & $\mathrm{FV}(\mathrm{mol} / \mathrm{h})$ \\
\hline $\mathrm{CO}_{2}$ & 24287420.82 \\
$\mathrm{CO}$ & 106949.50 \\
$\mathrm{C}_{2} \mathrm{H}_{4}$ & 3125.39 \\
$\mathrm{CH}_{2} \mathrm{O}$ & 3114.58 \\
\hline
\end{tabular}

auxiliary power systems, ground support facilities, and ground transportation on the emission of the whole airport theoretically, and the computing model of the emission inventory is given.

Auxiliary power units are another operator besides the main engine which also is a big emission source. Moreover, the emission near the ground is not only the emission of the aircraft in the LTO phase but also the emissions of ground support equipment, such as baggage tractors, transfer vehicles, aircraft tractors, platform trucks, passenger cars, car ferry, power cars, air-conditioned car, and air vehicles. In addition, the airport has a large number of vehicle parking; there are quite a large number of emissions when the car is at startup which should not be ignored. Last but not the least, the data we used in method 2 are published by the IPCC; these data are only a default value of all airports in the world which means that they may be not very suitable for Beijing Capital Airport.

\section{Conclusions}

In this paper, the emission of Beijing Capital Airport was monitored in four seasons with SOF-FT-IR, and $\mathrm{CO}_{2}, \mathrm{CO}$, $\mathrm{C}_{2} \mathrm{H}_{4}$, and $\mathrm{CH}_{2} \mathrm{O}$ are selected as the target gases. In accordance with the analysis, it can be seen that the concentration distribution approximately appears to be in low concentration at the upwind side and high concentration at the downwind direction which is consistent with the physical truth. Several points that have high concentration existed at the upwind direction which is mainly because the uncertainty of wind velocity and direction and the bump or anything else happened in the monitoring vehicle. 
Comparing the data acquired with the SOF-FT-IR and that acquired with method 2 published by the IPCC, we see that the former is higher than the latter. The main reason is that method 2 only estimates the emission of the main engine of the aircraft while the SOF-FT-IR measures the emission of the entire airport; there are so many emission sources such as auxiliary power plant emission sources, ground support equipment, ground transportation sources, and other stationary sources. The emissions of all these devices are not ignored apart from those of the aircrafts. It is also easy to find that even in the airport that is as large as Beijing Capital Airport, the emission of the aircraft only occupies a little part of the whole airport. The data are of great value for the government to evaluate and adopt appropriate policy to improve the air quality. The results measured by SOF-FT-IR also can be used to analyze the contribution of the emissions of the whole airport to air pollution quantitatively. Besides, the concentration distribution also can be used to locate the emission sources immediately as soon as the emission exceeds the threshold set by the government.

The mobile SOF-FT-IR can be used to monitor the emission of the surface source and provided a versatile, efficient, and robust tool to improve the statistics of emission of VOCs and study emissions of other gases and variations in emissions with temperature, in different seasons. What is more, the instrument is complementary to other techniques used to quantify emissions, such as in suit measurements or stationary instruments. Last but not the least, with the SOFFT-IR, a source can be isolated and quantified remotely.

\section{Conflicts of Interest}

The authors declare that they have no conflicts of interest.

\section{Acknowledgments}

The authors gratefully acknowledge the weather monitoring station in Beijing Capital Airport for their support information and the financial support by the National Natural Science Foundation of China (Grant no. 41605015) and Director Fund of Anhui Institute of Optics and Fine Mechanics (Grant no. AGHH201502). This project was supported by the National Natural Science Foundation of China (Grant nos. 41605015 and 41375027) and Director Fund of Anhui Institute of Optics and Fine Mechanics (Grant no. AGHH201502).

\section{References}

[1] L. Hui, "The Sky's Limit for Aircraft Emissions," World Environment, vol. 6, pp. 61-62, Ministry of Environmental Protection Mission Center, Beijing, China, 2006.

[2] A. Macintosh and L. Wallace, "International aviation emissions to 2025: can emissions be stabilised without restricting demand?," Energy Policy, vol. 37, no. 1, pp. 264-273, 2009.

[3] F. Lu, "China Large Aircraft Strategy Research Report," Business Week, vol. 6, pp. 29-49, 2005.

[4] IPCC, 2006 IPCC Guidelines for National Greenhouse Gas Inventories, Part II, Energy, Switzerland, 2006.
[5] C. Wang, Research on Evaluation Theory and Simulation Application of Flight Procedure Operation, Nanjing University of Aeronautics and Astronautics, Nanjing, 2012.

[6] J. G. David, E. P. Joyce, and H. L. David, "Aviation and the global atmosphere," Technical Report, Intergovernmental Panel on Climate Change (Cambridge University Press), Cambridge, 1999.

[7] J. S. Kurniawan and S. Khardi, "Comparison of methodologies estimating emissions of aircraft pollutants, environmental impact assessment around airports," Environmental Impact Assessment Review, vol. 31, no. 3, pp. 240-252, 2011.

[8] Y. Zhou and S. J. Fan, "Review and progress research on the impacts of pollutant emissions on atmospheric environment for aircraft take-off and landing activities," Meteorological and Environmental Sciences, vol. 36, pp. 62-67, 2013.

[9] D. L. Albritton and G. T. Aamanatidis, "Global atmospheric effects of aviation: report of the proceedings of the symposium," 1997.

[10] S. B. Fan, L. Nie, and X. F. Li, "Calculating airport emission with EDMS model," Journal of Safety and Encironment, vol. 10, pp. 93-96, 2010.

[11] U. Kesgin, “Aircraft emissions at Turkish airports," Energy, vol. 31, no. 2-3, pp. 372-384, 2006.

[12] K. N. Yu, Y. P. Cheung, T. Cheung, and R. C. Henry, "Identifying the impact of large urban airports on local air quality by nonparametric regression," Atmospheric Environment, vol. 38, no. 27, pp. 4501-4507, 2004.

[13] G. Liu, H. Wang, and P. Lu, "Gas Turbine Engine Emissions Measurement," Journal of Aerospace Power, vol. 18, pp. 348352, 2003.

[14] K. Schäfer, C. Jahn, P. Sturm, B. Lechner, and M. Bacher, "Aircraft emission measurements by remote sensing methodologies at airports," Atmospheric Environment, vol. 37, no. 37, pp. 5261-5271, 2003.

[15] M. L. Gao, Study on Multi-Component Gases Quantitative Analysis Based on FT-IR, University of Science and Technology of China, Hefei, 2010.

[16] "Beijing Capital International Airport Co., Ltd.," http://www. bcia.com.cn/investor/operation-affiche.shtml.

[17] H. Peace, J. Maughan, B. Owen, and D. Raper, "Identifying the contribution of different airport related sources to local urban air quality," Environmental Modelling \& Software, vol. 21, no. 4, pp. 532-538, 2006.

[18] Q. Xia, Research on the Atmospheric Environment Impact Assessment from Aircraft Engine Emissions in Airport, Nanjing University of Aeronautics and Astronautics, Nanjing, 2009. 

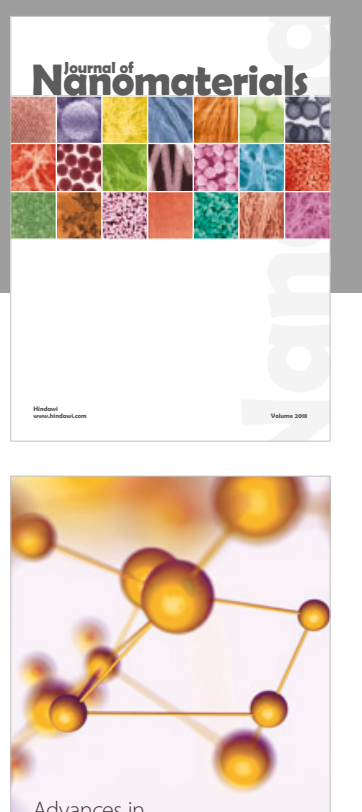

Physical Chemistry
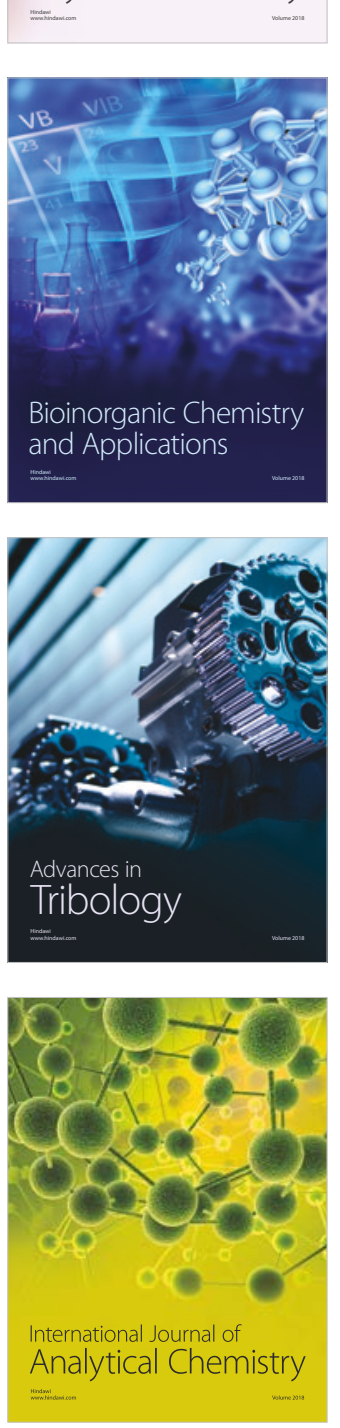

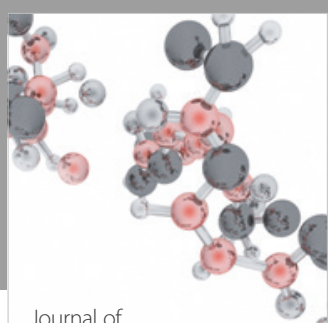

Analytical Methods

in Chemistry

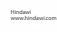

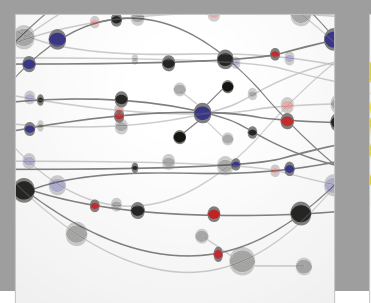

The Scientific World Journal

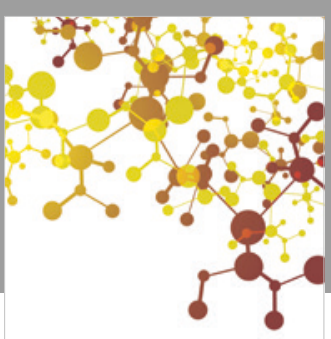

Journal of

Applied Chemistry
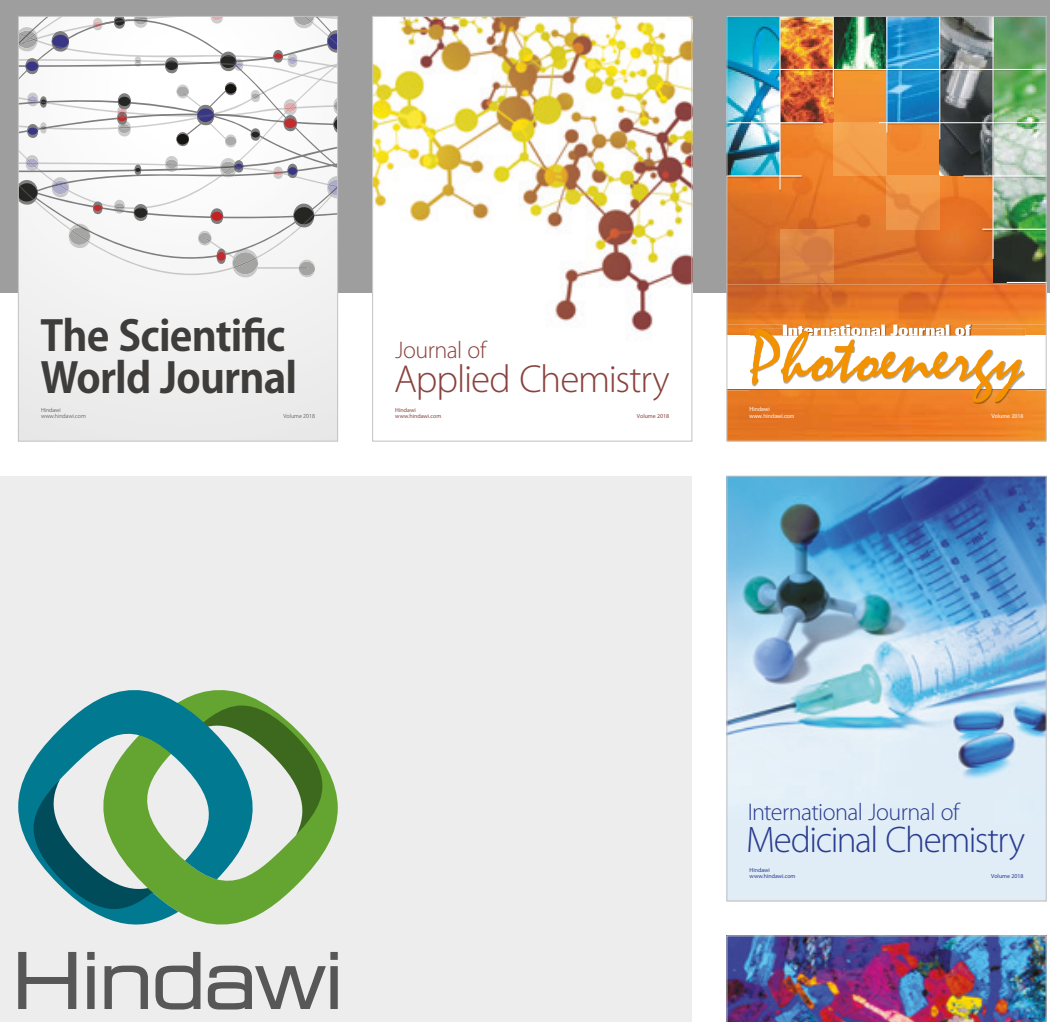

Submit your manuscripts at

www.hindawi.com
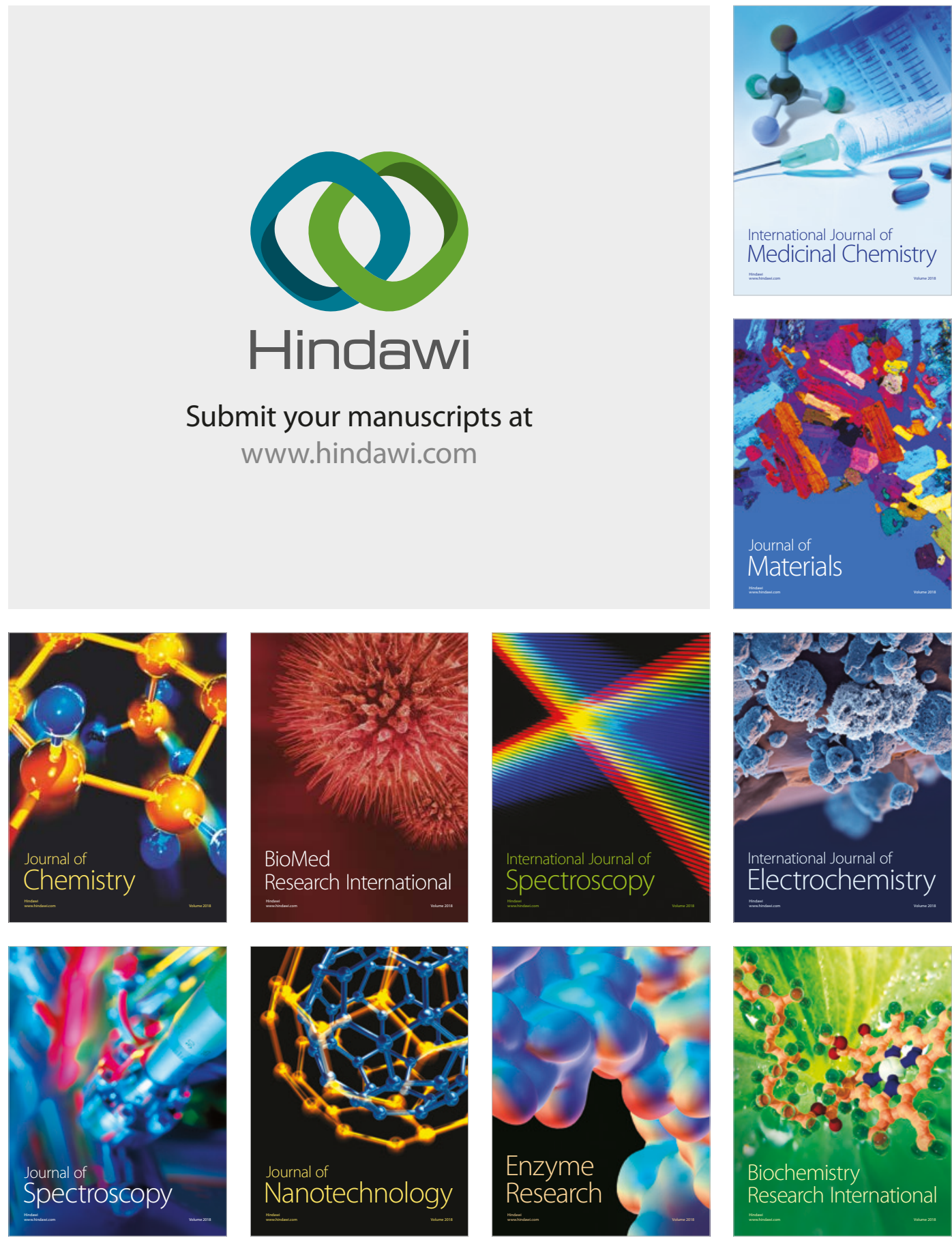
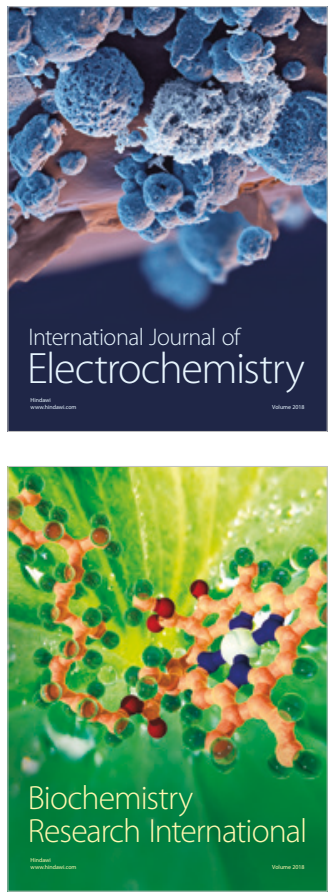